\title{
The PERSEO Experience: A Water-Filled Garment Prototype for Personal Radiation Protection of Astronauts Successfully Tested on Board the International Space Station
}

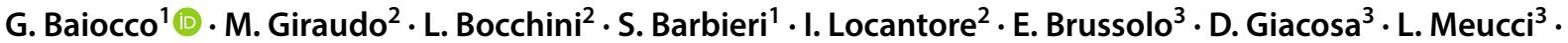 \\ S. Steffenino ${ }^{3} \cdot$ A. Ballario ${ }^{4}$ - B. Barresi ${ }^{4} \cdot$ R. Barresi ${ }^{4} \cdot$ M. Benassai ${ }^{5} \cdot$ L. Ravagnolo ${ }^{5} \cdot$ L. Narici $^{6,7} \cdot$ A. Rizzo ${ }^{6,7}$. \\ E. Carrubba ${ }^{8} \cdot$ F. Carubia ${ }^{8}$. G. Neri ${ }^{8} \cdot$ M. Crisconio ${ }^{9} \cdot$ S. Piccirillo ${ }^{9} \cdot$ G. Valentini ${ }^{9}$. S. Barbero ${ }^{10} \cdot$ M. Giacci $^{10}$. \\ C. Lobascio ${ }^{2} \cdot$ A. Ottolenghi ${ }^{1}$
}

Received: 25 February 2020 / Revised: 3 May 2020 / Accepted: 7 May 2020 / Published online: 20 May 2020

(c) The Author(s) 2020

\begin{abstract}
The PERSEO project (PErsonal Radiation Shielding for intErplanetary missiOns), funded by the Italian Space Agency, has led to the development of a first technological demonstrator of a radiation shielding garment, to be used in a pressurized space habitat, that can be filled at need with on-board water and used for personal protection in case of solar particle events. The collaboration, including academic partners and companies active in space research and technology development, designed and manufactured the prototype that has been successfully tested on board the International Space Station by the European Space Agency astronaut Paolo Nespoli in November 2017, during the VITA mission. The effectiveness of the garment in terms of reduction of the radiation dose to sensitive organs (subject to the occurrence of short-term non-cancer effects following acute exposure) has been evaluated with Monte Carlo simulations with an anthropomorphic phantom. The successful outcome of the experimental session on board has demonstrated the practicality of use and wearability of the prototype, and, in perspective, the feasibility of a personal radiation shielding strategy, complementary to habitat shielding and based on the use of available resources, of fundamental importance also in view of future manned interplanetary missions.
\end{abstract}

Keywords Space radiation protection · Shielding spacesuit · Resource optimization · International Space Station

G. Baiocco

giorgio.baiocco@unipv.it

1 Physics Department, University of Pavia, Via Bassi 6, 27100 Pavia, Italy

2 Thales Alenia Space-Italy, Turin, Italy

3 Società Metropolitana Acque Torino S.P.A., Turin, Italy

4 AVIOTEC S.P.A., Turin, Italy

5 ALTEC S.P.A., Turin, Italy

6 Physics Department, University of Rome Tor Vergata, Rome, Italy

INFN-Roma2, Rome, Italy

8 Kayser Italia S.R.L., Livorno, Italy

9 Italian Space Agency, Rome, Italy

10 ARESCOSMO S.P.A., Caselle Torinese, Italy

\section{Introduction}

The PERSEO (PErsonal Radiation Shielding for intErplanetary missiOns) project, one of the VITA experiments funded and supervised by the Italian Space Agency (ASI), has been successfully conducted on board the International Space Station (ISS) by the European Space Agency (ESA) astronaut Paolo Nespoli (Expedition 52-53) in November 2017. ASI has been granted access to the ISS utilization resources in the framework of the Memorandum of Understanding (MoU) with NASA, and completed its sixth flight opportunity with the VITA mission. In this framework, ASI availed itself of the industrial support services provided by Kayser Italia for the new payload integration process, safety, operations and logistics towards NASA. The PERSEO collaboration, coordinated by the University of Pavia and including academic partners and companies active in space research and technological development, developed the first prototype of a water-filled garment, a technological demonstrator of 
a personal radiation protection device for astronauts, to be used in a pressurized space habitat in case of occurrence of solar particle events. The prototype was designed and manufactured in compliance with safety requirements and all requirements for use in a space habitat, completing all necessary ground-verification tests. During the experimental session on board, Paolo Nespoli successfully tested the filling of the garment with ISS water, wore the garment for $\sim 30$ min verifying its comfort, and later drained the water back in the on-board water recovery system without water waste, demonstrating the feasibility of a personal radiation protection strategy based on the use of resources available in the space habitat. The efficacy of the prototype in terms of achievable dose reduction to organs subject to the insurgence of short-term non-cancer effects has been evaluated based on Monte Carlo simulations with an anthropomorphic phantom, exposed to reference solar proton spectra in low-shielding conditions, as it could happen in an emergency scenario during a deep-space mission. Dedicated measurements at the TIFPA (Trento Institute for Fundamental Physics and Application) proton accelerator facility have also been conducted to benchmark simulation results. For a complete report on the project see [1].

\section{Countermeasures to Solar Particle Events}

Transition to a new spacesuit design gives an opportunity to optimize the next generation spacesuit for reduced potential health effects during an accidental exposure to space radiation $[2,3]$. The PERSEO garment has been particularly conceived to protect astronauts in case of a Solar Particle Event (SPE): SPEs are hardly predictable events, during which a large amount of particles (mainly protons) are injected from the Sun into interplanetary space and impact on the space habitat. Solar protons are of low energy (up to few hundreds of $\mathrm{MeV}$ ) with respect to galactic cosmic rays-the other main component of the space radiation environment-and a radiation protection strategy based on passive shielding is successful: at present, in case of SPE alert, astronauts are required to take shelter in dedicated areas of the habitat, with increased wall thickness. Nevertheless, also in view of future higher-complexity scenarios for deep-space missions, habitat shielding cannot be the ultimate solution: there might be the need to exit the radiation shelter and to operate in lowershielded areas of the habitat when the event is in progress, or the risk of being caught by (or alerted for) a SPE without being able to immediately get to the radiation shelter. Therefore, innovative solutions based on personal shielding have to be envisaged, to avoid the onset of immediate consequences for the astronauts' health, as those following an acute radiation exposure to doses higher than organ-dependent thresholds. In particular, it is of utmost importance to protect blood forming organs (BFO), as the lethality of an acute exposure can be mainly attributed to heavy damages to the hematopoietic system. Among possible solutions, those making use of multi-functional resources already available in the space habitat have to be preferred, thus avoiding the costs and logistic constraints of adding extra material solely for shielding purposes to the mission payload. Water is an excellent candidate material, as it is a necessary on-board resource with good shielding properties, provided that its use for shielding does not lead to its waste $[4,5]$.

\section{The Garment Prototype: Development, Test on the ISS and Shielding Efficacy}

\subsection{The PERSEO Garment Prototype}

The prototype (Fig. 1a) is a sleeveless Nomex ${ }^{\circledR}$ Garment with 4 embedded water containers made of Coretech ${ }^{\circledR}$ (Saint-Gobain), all connected by a circuitry system with valves and a single interface to allow for use of ISS water resources. The garment is fillable with $\sim 22 \mathrm{~L}$ of water, and, when filled, water bags reach a thickness of $\sim 7 \mathrm{~cm}$. The lateral dimensions of the bags have been tailored on the astronaut's torso: bags protecting the astronaut's back are $37 \times 37 \mathrm{~cm}^{2}$, while bags protecting the upper chest and abdomen are $25 \times 25 \mathrm{~cm}^{2}$. In compliance with safety requirements to prevent water leakage when filling, bags were designed to resist high differential pressure (24 psig) at the filling interface without leakage or rupture. Structural analysis (with LS-DYNA) and dedicated ground-verification tests have been conducted to verify this requirement. As the water dispenser system indicated for use by NASA delivers iodinated water, break strength measurements after immersion tests have also been conducted to verify the compatibility of Coretech ${ }^{\circledR}$ with iodinated water, and choose the appropriate size of welded joints used for manufacturing the bags. The release by the material of hazardous substances of any kind in the water (both volatile organic compounds and polycyclic aromatic hydrocarbons, for all parameters subject to water primary drinking regulations) has also been excluded. Bags in the integrated configuration also underwent a series of soaking and rinsing steps, to verify the appropriate cleanliness requirement before filling (particulate counts in size classes and the absence of non-volatile residue). Finally, the system was successfully tested for endurance to vacuum.

On August 14, 2017, the PERSEO payload (ad-hoc manufactured Nomex ${ }^{\circledR}$ transport bag containing the vacuumed PERSEO garment) was successfully launched within the Dragon spacecraft with SpaceX twelfth commercial resupply services mission, from NASA Kennedy Space Center, Florida. Dragon was captured and successfully docked to the ISS 2 days later, on August 16 [1]. 
Fig. 1 The PERSEO garment prototype, a during pre-flight inspection at Johnson Space Center (JSC)-Houston, USA; b worn by ESA astronaut Paolo Nespoli during the experimental session on board the ISS

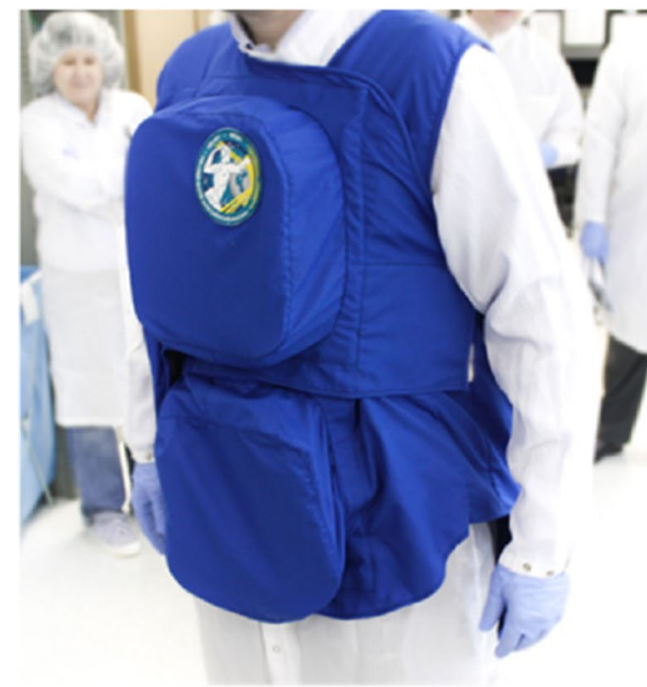

(a)

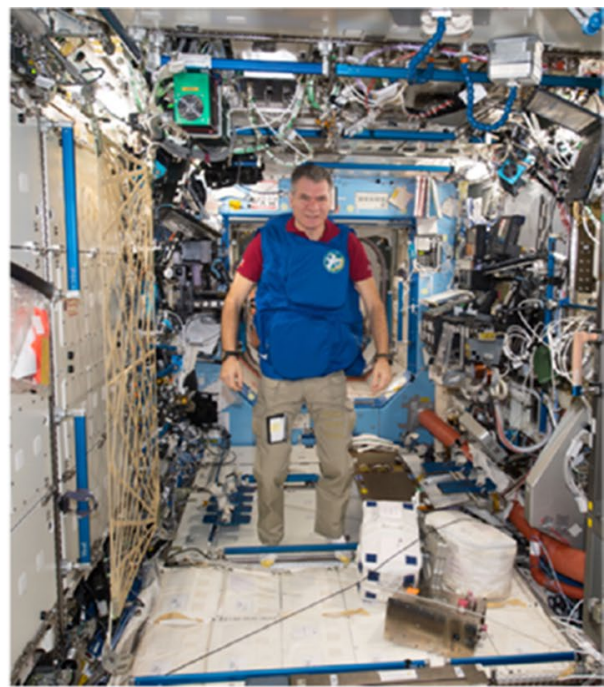

(b)

\subsection{The Experimental Session on Board the ISS}

The PERSEO session on board the ISS took place on November 7, 2017. The scientific objectives of the experimental session have been fully achieved and the astronaut carried out on-board operations as planned, with no deviation from the procedures. Operations were followed realtime both from NASA mission control center and from the Italian team of developers. The outcome of the session has been evaluated based on analysis of pictures and video recording and on the feedback collected from the astronaut in a dedicated questionnaire and in a post-mission debrief session with the developers. The filling ( $20 \mathrm{~min})$ and draining ( $40 \mathrm{~min})$ of the garment have been judged extremely easy to perform, with no water waste, the duration of such phases being dictated by the characteristics of the water filling/draining interfaces indicated for use by NASA. Once filled, the astronaut wore the garment for $\sim 30 \mathrm{~min}$ (Fig. 1b), judging it comfortable enough, not significantly hindering his movements and not posing major problems because of increased inertial mass. As minor discomfort, he reported a slight feeling of cold (due to the temperature of on-board water used for the filling being lower than body temperature), which suggests the implementation of a thermic insulation for the bags, and the possibility of displacement of bags on the torso, which suggest the implementation of further tightening systems [1].

\subsection{Shielding Effectiveness}

We performed GRAS-Geant4 Monte Carlo simulations with an anthropomorphic phantom, exposed to different reference solar proton spectra with and without a simplified software replica of the PERSEO garment. The phantom is placed in an $\mathrm{Al}$ module, whose wall thickness is varied in the range $1.5-10 \mathrm{~cm}$, to mimic exposures in different shielding conditions. As main indicator of the garment shielding effectiveness, we take the dose reduction to blood forming organs, taking into account the importance of different bone structures in terms of their content of red bone marrow, the tissue actively producing blood cells. Calculations in this simplified simulation setup give a dose reduction value to BFO of $42 \pm 3 \%$ for the lowest-shielded condition, which is decreased to $\sim 30 \%$ in case of thicker shielding, when habitat walls are themselves responsible for the stopping and/or slowing down of a part of the spectrum of incoming solar protons. Dose reduction results can be translated in terms of increase of the time interval before a given dose threshold for the onset of health effects is reached: such dose limit is set to 250 mGy-Eq (physical dose in mGy multiplied by a relative biological effectiveness weight of 1.5 for protons) by NASA for BFO. Considering a peak dose rate of $\sim 100 \mathrm{mGy}-$ $\mathrm{Eq} / \mathrm{h}$ for an astronaut caught by a SPE during intra-vehicular activities in a low-shielded area of the habitat, the BFO dose limit would be reached in $\sim 2.5 \mathrm{~h}$ if the astronaut is not wearing any personal personal protection device, or in almost double of this time if he/she is wearing a garment similar to the PERSEO prototype. For all details see [1] and references therein.

\section{Concluding Remarks}

The successful outcome of the PERSEO project demonstrates the feasibility of personal radiation shielding using on-board water as a strategy complementary to habitat shielding for future deep-space human exploration missions. The characteristics of the first PERSEO technological 
demonstrator are the results of a wide variety of constraints (human factors, safety requirements and limitations of available resources) and requirements, not least those dictated by the specific characteristics of the interfaces for water delivery/recovery on board the ISS, indicated by NASA for use in this experimental session. For the development of future models to be used in different space habitats, water interfaces with appropriate characteristics should be selected/designed. This would certainly allow the design and manufacturing of water containers with more ergonomic shapes, while for this prototype a regular "parallelepiped" shape was the preferred option, mainly because of the requirement of resistance to a high differential pressure at filling. This would lead to a sure improvement of the garment wearability, that has been already judged positively for this first prototype. A new water delivery system could also be thought as faster and allowing the filling of multiple garments at the same time. Alternative strategies could also be explored: different garments could be kept filled at all time, used for water storage and ready to use for personal radiation protection in case of emergency, if the material in contact with water is verified to keep water quality unaltered. Also, water purification methods (not necessarily only iodine-based) should always be considered, in terms of compatibility with the chosen material. If the same concept of a garment with embedded protection elements is further explored, the same elements could be thought as water-storage devices, to be extracted from the garment and used to increase wall shielding at need when the garment is not in use. Finally, in perspective, new developments could address the use of shielding garments in the different operational contexts of interplanetary missions, with different conditions of gravity, temperature and pressure, including extravehicular activities and expanding the protection to other body areas.

Concluding, the know-how acquired by the PERSEO collaboration in the course of the project sets a solid basis for the developments of new-generation space radiation shielding garments for use in pressurized space habitats. PERSEO paved the way for the consolidation of personal radiation shielding using available resources as a fundamental strategy in view of future interplanetary missions as the one to Mars [1].

Acknowledgements PERSEO was funded by the Italian Space Agency (ASI) and coordinated by the University of Pavia (UniPv). Project partners: Thales Alenia Space-Italy (TAS-I) S.p.A.; Società Metropolitana Acque Torino (SMAT) S.p.A.; AVIOTEC S.p.A.; Advanced Logistic Technology Center (ALTEC) S.r.1.; University of Rome Tor Vergata (UniRMToV). Project support: Kayser Italia (KI) S.r.l. Project supplier: ARESCOSMO S.p.A. Acknowledgements are due to: all contributing scientists and staff members of the Italian Space Agency and of the project partners; to the payload integration manager from The Boeing Company; to the numerous NASA scientists and officers who advised and guided the implementation of the experiment on board the ISS; finally, to ESA astronaut Paolo Nespoli, for useful advices during the design and realization phase of the PERSEO garment, and for carrying out the experimental session on board the ISS. Thanks are due also to the whole crew of Expedition 52/53 for their support during on-board operations.

Funding PERSEO was funded by the Italian Space Agency (ASI), contract number 2016-3-U.0.

Availability of Data and Materials Data are available upon reasonable request to the corresponding author.

\section{Compliance with Ethical Standards}

Conflict of interest On behalf of all authors, the corresponding author states that there is no conflict of interest.

Code Availability GRAS-Geant 4 is now available from the ESA Space Software Repository (ESSR) at https://essr.esa.int/project/gras-geant 4-radiation-analysis-for-space as open source tool, under the "European Space Agency Community License-v2.3 Strong Copyleft”.

Open Access This article is licensed under a Creative Commons Attribution 4.0 International License, which permits use, sharing, adaptation, distribution and reproduction in any medium or format, as long as you give appropriate credit to the original author(s) and the source, provide a link to the Creative Commons licence, and indicate if changes were made. The images or other third party material in this article are included in the article's Creative Commons licence, unless indicated otherwise in a credit line to the material. If material is not included in the article's Creative Commons licence and your intended use is not permitted by statutory regulation or exceeds the permitted use, you will need to obtain permission directly from the copyright holder. To view a copy of this licence, visit http://creativecommons.org/licenses/by/4.0/.

\section{References}

1. Baiocco, G., et al.: A water filled garment to protect astronauts during interplanetary missions tested on board the ISS. Life Sci. Space Res. 18, 1-11 (2018)

2. Chancellor, J.C., et al.: Space radiation: the number one risk to astronaut health beyond low earth orbit. Life 4(3), 491-510 (2014)

3. Wilson, J., Anderson, B.M., Cucinotta, F.A., Ware, J., Zeitlin, C.J.: Spacesuit radiation shield design methods. SAE Tech. Paper 1, 2110 (2006). https://doi.org/10.4271/2006-01-2110

4. Vuolo, M., Baiocco, G., et al.: Exploring innovative radiation shielding approaches in space: a material and design study for a wearable radiation protection spacesuit. Life Sci. Space Res. 15, 69-78 (2017)

5. H. Wu et al.: Risk of acute radiation syndromes due to solar particle events in "Human Health and Performance Risks of Space Exploration Missions: Evidence Reviewed by the NASA Human Research Program". US National Aeronautics and Space Administration, pp. 171-190 (2010).

Publisher's Note Springer Nature remains neutral with regard to jurisdictional claims in published maps and institutional affiliations. 